\title{
ORIGINAL ARTICLE Successful pharmacotherapy for the treatment of severe feeding aversion with mechanistic insights from cross-species neuronal remodeling
}

\author{
WG Sharp ${ }^{1,2}$, AG Allen ${ }^{1,3}$, KH Stubbs ${ }^{2}$, KK Criado ${ }^{1,2}$, R Sanders ${ }^{4}$, CE McCracken ${ }^{1}$, RG Parsons ${ }^{5}$, L Scahill ${ }^{1,2}$ and SL Gourley $^{1,2,3,4}$
}

\begin{abstract}
Pediatric feeding disorders affect up to $5 \%$ of children, causing severe food intake problems that can result in serious medical and developmental outcomes. Behavioral intervention (BI) is effective in extinguishing feeding aversions, and also expert-dependent, time/labor-intensive and not well understood at a neurobiological level. Here we first conducted a double-blind, placebo-controlled trial comparing BI with BI plus D-cycloserine (DCS). DCS is a partial N-methyl-D-aspartate (NMDA) receptor agonist shown to augment extinction therapies in multiple anxiety disorders. We examined whether DCS enhanced extinction of feeding aversion in 15 children with avoidant/restrictive food intake disorder (ages 20-58 months). After five treatment days, BI improved feeding by $37 \%$. By contrast, BI+DCS improved feeding by $76 \%$. To gain insight into possible mechanisms of successful intervention, we next tested the neurobiological consequences of DCS in a murine model of feeding aversion and avoidance. In mice with conditioned food aversion, DCS enhanced avoidance extinction across a broad dose range. Confocal fluorescence microscopy and threedimensional neuronal reconstruction indicated that DCS enlarged dendritic spine heads-the primary sites of excitatory plasticity in the brain-within the orbitofrontal prefrontal cortex, a sensory-cognition integration hub. DCS also increased phosphorylation of the plasticity-associated extracellular signal-regulated kinase 1/2. In summary, DCS successfully augments the extinction of food aversion in children and mice, an effect that may involve plasticity in the orbitofrontal cortex. These results warrant a larger-scale efficacy study of DCS for the treatment of pediatric feeding disorders and further investigations of neural mechanisms.
\end{abstract}

Translational Psychiatry (2017) 7, e1157; doi:10.1038/tp.2017.126; published online 20 June 2017

\section{INTRODUCTION}

Pediatric feeding disorders involve chronic and severe problems with food consumption beyond ordinary developmental variations in hunger, food preferences and/or interest in eating. ${ }^{1}$ Children with this level of feeding difficulty fall under the broader psychiatric diagnosis of avoidant/restrictive food intake disorder, characterized by persistent failures to meet appropriate nutritional or energy needs resulting in faltering growth and/or significant nutritional deficiencies. ${ }^{2}$ Feeding problems of this magnitude affect as many as $5 \%$ of children and may contribute to serious medical and developmental outcomes. ${ }^{1,3}$ In addition to growth retardation, these outcomes may include invasive medical procedures (for example, feeding tube placement), ${ }^{4}$ compromised immune functioning, and/or impaired cognitive and emotional development. $^{5}$

Chronic food refusal often follows early medical problems, such as congenital or acquired respiratory, cardiac and gastrointestinal problems that cause difficult or painful eating experiences. ${ }^{1,4,6}$ However, resolution of the underlying medical problem often does not in and of itself coincide with improved oral intake. Frequent, intense and disruptive mealtime behaviors may be well engrained and serve to maintain the feeding disorder. ${ }^{7}$ To date, intensive behavioral intervention (BI) at multidisciplinary day treatment or inpatient hospital programs is the most empirically supported treatment for pediatric feeding disorders., ${ }^{1,8,9}$
Treatment typically involves 'escape extinction', during which a therapist persists with bite presentations to promote contact with food while ignoring problem behaviors. ${ }^{1,7}$ Unfortunately, when available, effective treatment programs are expensive, with costs as high as $\$ 50000$ per child for 40 days of intervention. ${ }^{10}$ Adjunctive treatments that could hasten time to effect would reduce the high cost.

D-cycloserine (DCS) is a partial $\mathrm{N}$-methyl-D-aspartate (NMDA) receptor agonist that augments extinction therapies in multiple contexts. ${ }^{11,12}$ These include treatment of acrophobia, ${ }^{13}$ social phobia, $^{12}$ obsessive-compulsive disorder ${ }^{14}$ and panic disorder, ${ }^{15}$ including in children. ${ }^{16}$ In a meta-analysis of nonhuman and human trials, Norberg et al. ${ }^{17}$ reported that the benefits of DCS augmentation were greatest early in treatment. These findings are parsimonious with evidence in animal models that DCS facilitates extinction conditioning through rapid memory consolidation processes. ${ }^{18,19}$

To our knowledge, there are no studies that combine pharmacological augmentation with $\mathrm{BI}$ in the treatment of pediatric feeding disorders. The purpose of this randomized, double-blind, placebo-controlled study was to examine the feasibility and preliminary efficacy of combining DCS with our standard BI for young children with chronic and severe food refusal. In tandem, we developed an animal model of severe feeding refusal and assessed the utility of DCS in facilitating avoidance extinction.

\footnotetext{
${ }^{1}$ Department of Pediatrics, Emory University School of Medicine, Atlanta, GA, USA; ${ }^{2}$ Pediatric Psychology and Feeding Disorders Program, The Marcus Autism Center, Atlanta, GA, USA; ${ }^{3}$ Yerkes National Primate Research Center, Atlanta, GA, USA; ${ }^{4}$ Department of Psychiatry, Emory University School of Medicine, Atlanta, GA, USA and ${ }^{5}$ Graduate Program in Integrative Neuroscience and Program in Neuroscience, Department of Psychology, Stony Brook University, Stony Brook, NY, USA. Correspondence: Dr WG Sharp, Pediatric Psychology and Feeding Disorders Program, The Marcus Autism Center, 1920 Briarcliff Road, Atlanta, GA 30329, USA.

E-mail: wgsharp@emory.edu
}

Received 11 April 2017; accepted 20 April 2017 
We used mutant mice expressing yellow fluorescence protein to allow for postmortem dendritic spine imaging and threedimensional reconstruction of individual spines, the primary sites of excitatory neurotransmission in the brain. We focused on the orbitofrontal cortex (oPFC), innervated by viscero-sensory structure $\mathrm{s}^{20}$ and also the basal amygdala, a primary site of aversive conditioning and its extinction. ${ }^{18,21}$

\section{MATERIALS AND METHODS}

\section{Clinical investigation}

This was a double-blind, placebo-controlled study of DCS augmentation in children receiving $\mathrm{BI}$ at a day treatment, pediatric feeding disorders program. ${ }^{22}$ The study occurred between April 2013 and September 2013. The trial concluded once we achieved our recruitment goal of 16 participants. This sample size was deemed sufficient to evaluate feasibility (that is, recruitment, retention, safety) of this pilot study while recognizing limitations to detect only large group differences.

Participants were randomly assigned to (i) daily $\mathrm{BI}+\mathrm{DCS}$ or (ii) daily $\mathrm{BI}$ +placebo. Randomization was completed by an Investigational Drug Service pharmacist using a computer software program, which assigned subjects to condition using permuted blocks. The Emory University Institutional Review Board approved the study protocol, and parents provided written informed consent. This study was registered with Clinicaltrials.gov.

Setting and subjects. The male and female subjects had to be 18 months to 6 years old, deemed appropriate for extinction-based feeding intervention based on active and persistent food refusal (severe tantrums, disruptive behavior) that severely restricted the volume of food consumed during meals, and meet diagnostic criteria for avoidant/restrictive food intake disorder as evidenced by dependence on enteral feeding or oral nutritional formula supplementation. ${ }^{2}$ We selected this age range because chronic food refusal is most commonly observed in early childhood, which is consistent with recent summaries of the treatment literature. ${ }^{1,8}$ Inclusion criteria also required history of a significant medical condition, such as gastroesophageal reflux disease, esophagitis or food allergy, associated with the development of the feeding problem. Exclusion criteria included (i) prior extinction-based feeding intervention; (ii) anatomical/active medical problems prohibiting safe oral intake; (iii) taking medications contraindicated for DCS; and (iv) history of medication refusal via formula for children reliant on oral supplementation. A child psychiatrist assessed the medical status before enrollment and monitored adverse events during the study.

BI treatment. Meals occurred in treatment rooms equipped with adjacent observation rooms. Treatment occurred across five consecutive days involving 15 total meals divided into five-bite sessions. A team of four behavioral psychologists with expertise in pediatric feeding disorders and two trained therapists conducted the first 13 treatment meals. The caregivers were trained on the protocol during meal 14 . To promote generalization, the caregiver served as the primary feeder for meal 15 . We conducted a parent-led meal (without medication) in the clinic at 1-month post treatment.

The BI treatment manual incorporated escape extinction and reinforcement procedures., ${ }^{1,22}$ During each meal, the feeder (that is, therapist or parent) presented one item from each food group using a standardized schedule (four foods per meal). The menu drew from a pre-established set of eight foods selected by a registered dietician with low probability for food allergies or cultural/parental dietary restrictions. The first treatment meal involved six sessions of a structured meal protocol involving standardized instructions ('Take a bite'), meal length (30 min) and bite volume $(1 \mathrm{ml})$. The child remained seated in a high chair throughout structured meal protocol and the feeder presented bites every $30 \mathrm{~s}$. The feeder, however, removed individual bite presentations in response to disruptive behavior (pushing away the spoon; head turns). This baseline condition provided a further screen for active and persistent food refusal appropriate for extinction. The feeder introduced escape extinction in the form of non-removal of the spoon during the second meal. Non-removal of the spoon involved placing the spoon at the child's lips, following the lips with the spoon in response to head turning, ignoring disruptions and depositing the bite immediately once the mouth is open., 23,24 Nonremoval of the spoon remained in place for all remaining meals.
DCS treatment. As in previous trials in adults ${ }^{17}$ and children, ${ }^{16}$ DCS was prepared by the Investigational Drug Service pharmacist using Seromycin $250 \mathrm{mg}$ capsules. The dose was $0.7 \mathrm{mg} \mathrm{kg}^{-1}$. Lactose monohydrate NF powder was added to ensure equal amounts of powder in each drug vial. Placebo doses used lactose monohydrate NF powder alone. Parents administered DCS or placebo $1 \mathrm{~h}$ prior $( \pm 10 \mathrm{~min})$ to the first treatment meal per day via their child's typical method of medication administration (g-tube, syringe, cup/bottle). Study staff observed medication administration. We utilized a single daily dose based on prior research suggesting a lower risk of adverse effects compared to multiple daily dosing. ${ }^{16}$ With the exception of the Investigational Drug Service pharmacist, all involved (that is, researchers, therapists, parents, subjects) were blind to the treatment condition until study completion.

Clinical measures. Outcome measures were rapid swallowing and disruptions. Rapid swallowing was defined as swallowing the entire bolus within $30 \mathrm{~s}$ after the bite presentation. The feeder visually confirmed this behavior using a three-step prompting sequence (verbal, model, physical) to look inside the mouth. Disruptions were defined as turning the head 45 degrees away from the spoon and/or pushing away the spoon or feeder's hand/arm during a bite presentation. Counts of each behavior were converted into percentages by dividing the occurrences of the target behavior by the total bites presented. A second observer rated $38 \%$ of meals to check inter-observer agreement. The mean kappa was 0.87 for rapid swallowing (range: $0.78-1.0$ ) and 0.76 for disruptions (range: $0.65-$ 1.00 ), indicating substantial agreement. ${ }^{25}$

Statistical analyses. We used SAS 9.3 for Windows (Cary, NC, USA) for all statistical analyses, assessing significance at the 0.05 level unless otherwise noted. Before analysis, all outcome measures were assessed for normality using histograms, normal probability plots and the Anderson-Darling test for normality. For variables where normality was suspect, analysis was carried out using the ranks of the data, by replacing the actual data values with their rank in the data set (that is, their relative position in the data set). Mixed-effect regression models were used to assess the effect of study group (drug vs placebo) and the effect of meal number on each outcome measure while controlling for the correlation between meals. Models included a random intercept. Time and treatment were treated as fixed effects. Because of the unequal variances, the Kenward-Roger approximation to the degrees of freedom was applied and the covariance structures were modeled separately for drug and placebo groups when group variances were heteroskedastic. Unstructured covariance matrices were used when feasible to allow the errors of the dependent observations to be correlated and variances to differ across time. When an unstructured covariance matrix could not be fit, a first-order autoregressive was used instead. All models initially included the interaction between study group and meal number, but was subsequently removed from the model if it was not significant at the 0.10 level. Due to the small sample size and pilot nature of this project, no post hoc multiple procedure was utilized. We chose to include the within group comparison of meal 1 vs meal 15 for each treatment group. The presented $P$-values are unadjusted for multiple comparisons.

\section{Rodent investigation}

The mice were 61-day-old C57BL/6 females bred in-house from Jackson Labs (Bar Harbor, ME, USA) stock. For studies involving dendritic spine imaging, the mice expressed thy1-derived yellow fluorescence protein $(\mathrm{H}$ $l_{i n e^{26}}$ ) and were back-crossed onto a C57BL/6 background (Jackson Labs). The mice were maintained on a $12 \mathrm{~h}$ light cycle $(0700 \mathrm{~h}$ on) and provided food and water ad libitum except during testing, when body weights were maintained at $\sim 95 \%$ of baseline to motivate food-reinforced responding. The procedures were Emory University IACUC-approved.

DCS treatment. DCS (1.5-15 $\mathrm{mg} \mathrm{kg}^{-1}$, intraperitoneally, $1 \mathrm{ml}$ per $\left.100 \mathrm{~g}\right)$ dissolved in phosphate-buffered saline was prepared fresh daily.

Operant conditioning. The mice were first trained to nose poke for food pellets (20 mg each; Bioserv, Flemington, NJ, USA) using Med-Associates conditioning chambers. The training was initiated with a fixed ratio one schedule of reinforcement (that is, each response results in the delivery of one pellet). Daily sessions ended when 60 pellets were delivered or at $70 \mathrm{~min}$. In a final experiment, the mice were then shifted to a random interval $30 \mathrm{~s}$ schedule of reinforcement for four additional sessions. This schedule of reinforcement is used to promote inflexible, habit-based 
responding. ${ }^{27}$ Following every training session, the mice were returned to the home cage and sufficient chow (Lab Diet, St. Louis, MO, USA) was provided to ensure that body weights remained at $\sim 95 \%$ of each mouse's original free-feeding baseline.

Conditioned aversion. Next, the mice were allowed unlimited access in a clean cage to the food pellets used in the operant conditioning procedure. The mice were allowed to feed for $1.5 \mathrm{~h}$, and immediately following that the mice were injected with $0.15 \mathrm{M} \mathrm{LiCl}\left(4 \mathrm{ml}\right.$ per $100 \mathrm{~g}$, intraperitoneally ${ }^{28}$ ), inducing temporary gastric malaise and conditioned aversion. The mice were then fed $\geqslant 3 \mathrm{~h}$ later with regular chow to maintain healthy body weights. This procedure was repeated the following day. To confirm that conditioned aversion caused a reduction in responding for the food pellet (that is, avoidance), the mice were placed the following day in the conditioning chambers, and nose poking for 10 min in extinction was assessed. Except in the case of extended training, the conditioned aversion procedure was repeated if mice did not reduce their food intake during this period.

Avoidance extinction. Next, all the mice were returned to the conditioning chambers. One food pellet was provided non-contingently, and nose poking was reinforced for $25 \mathrm{~min}$. The mice were tested for three consecutive days, with DCS or vehicle treatment immediately following each session, targeting the consolidation of new learning (that is, 'the food pellet is no longer associated with sickness'). Unless otherwise indicated, three sessions without injection then followed to determine whether the effects of DCS were durable, persisting despite drug discontinuation, as occurred during the 1-month follow-up appointment in our clinical population. The nose-poke rates were normalized to the first session to account for individual differences between mice.

DCS vs vehicle groups were assigned by matching mice based on predrug response rates.

Phospho-ERK1/2 immunostaining. One cohort of mice $\left(1.5 \mathrm{mg} \mathrm{kg}^{-1}\right.$ group) was killed following the final test session by rapid decapitation. The brains were harvested and stored in 4\% paraformaldahyde for $48 \mathrm{~h}$ and then transferred to $30 \% \mathrm{w} / \mathrm{v}$ sucrose before being sectioned into $45 \mu \mathrm{m}$ sections. The sections were blocked in a phosphate-buffered saline solution containing $2 \%$ normal goat serum, $1 \%$ bovine serum albumin and $0.3 \%$ Triton X-100 (Sigma, St. Louis, MO, USA) for $1 \mathrm{~h}$ at room temperature. The sections were then incubated in primary antibody solution containing $0.3 \%$ normal goat serum, $1 \%$ bovine serum albumin and $0.3 \%$ Triton $\mathrm{X}-100$ at $4{ }^{\circ} \mathrm{C}$ for $48 \mathrm{~h}$. phospho-ERK1/2 (1:400; Cell Signaling, Danvers, MA, USA) served as the primary antibody. The sections were incubated in secondary antibody solution containing $0.5 \%$ normal goat serum and $0.3 \%$ Triton X-100, with Alexa Fluor 633 (1:200; Life Technologies, Carlsbad, CA, USA) serving as the secondary antibody.

The sections were imaged in a single session using a Nikon 4550s SMZ18 microscope with settings held constant. Fluorescence density was determined using ImageJ. A sampling area was drawn using a mouse brain atlas $^{29}$ as reference to confirm that samples were collected from equivalent rostral-caudal, medial-lateral and dorso-ventral positioning throughout. The sampling shape and area for each brain region were held constant across mice, then densitometry values were normalized to the size of the sampling area. Imaging and scoring were completed by a single rater blinded to group.

Dendritic spine imaging. One cohort of mice $\left(1.5 \mathrm{mg} \mathrm{kg}^{-1}\right.$ group) was killed following the final test session by rapid decapitation. Dendrites on deep-layer lateral oPFC neurons were imaged using fluorescence confocal microscopy and reconstructed in three dimensions using Imaris software. The methods are described elsewhere, ${ }^{30}$ except a Leica TSC SP8 microscope was used. Dendritic spines were classified according to ref. 31.

Between five and seven segments from secondary and tertiary dendritic branches within 50-150 $\mu \mathrm{m}$ of the soma were collected. Each mouse contributed single density values (mean per mouse) to statistical analyses to avoid artificial power inflation. Due to the stellate appearance of oPFC neurons, apical vs basal branches were not distinguished. ${ }^{32} \mathrm{~A}$ single rater scored all images and was blind to treatment condition until study completion.

Statistical analyses. Food intake, response rates and fluorescence densitometry were compared by two-tailed analysis of variance with repeated measures when appropriate. Dendritic spine densities were
Table 1. Summary of study participants

\begin{tabular}{|c|c|c|c|}
\hline Characteristic & $\begin{array}{l}\text { Overall } \\
(\mathrm{N}=15)\end{array}$ & $\begin{array}{l}\text { Drug } \\
(\mathrm{N}=8)\end{array}$ & $\begin{array}{l}\text { Placebo } \\
(\mathrm{N}=7)\end{array}$ \\
\hline $\begin{array}{l}\text { Age (in months), } \mathrm{M} \pm \text { s.d. } \\
\text { Gender-male, } N(\%)\end{array}$ & $32.7 \pm 10.6$ & $33.9 \pm 10.1$ & $31.3 \pm 12.5$ \\
\hline Gender-male, $N(\%)$ & $10(66.7)$ & $5(62.5)$ & $5(71.4)$ \\
\hline \multicolumn{4}{|l|}{ Feeding concerns, N (\%) } \\
\hline Feeding tube & $9(60.0)$ & $5(62.5)$ & $4(57.1)$ \\
\hline Bottle/liquid dependence & $6(40.0)$ & $3(37.5)$ & $3(42.9)$ \\
\hline \multicolumn{4}{|l|}{ Medical issues, by history ${ }^{\mathrm{a}}, \mathrm{N}(\%)$} \\
\hline $\begin{array}{l}\text { Gastroesophageal reflux } \\
\text { disease }\end{array}$ & $12(80.0)$ & $5(62.5)$ & $7(100)$ \\
\hline Food allergy & $5(33.3)$ & $2(25.0)$ & $3(42.9)$ \\
\hline Failure to thrive & $9(60.0)$ & $5(62.5)$ & $4(57.1)$ \\
\hline Heart problem/defect & $4(25.0)$ & $0(0.0)$ & $4(57.1)$ \\
\hline Aspiration & $2(13.3)$ & $1(6.2)$ & $1(14.3)$ \\
\hline Bronchopulmonary dysplasia & $2(13.3)$ & $2(25.0)$ & $0(0.0)$ \\
\hline \multicolumn{4}{|l|}{ Mealtime behavior problems ${ }^{\mathrm{b}}, \mathrm{N}(\%)$} \\
\hline $\begin{array}{l}\text { Turning head away the } \\
\text { spoon }\end{array}$ & $14(93.3)$ & 7 (87.5) & $7(100)$ \\
\hline Pushing away spoon & $15(100)$ & $8(100)$ & $7(100)$ \\
\hline Crying & $12(80.0)$ & $7(87.5)$ & $5(71.4)$ \\
\hline Leaving the table & $7(46.7)$ & $3(37.5)$ & $4(57.1)$ \\
\hline
\end{tabular}

compared by two-tailed Student's $t$-tests. Head volume cumulative density curves were compared by two-sample Kolmogorov-Smirnov tests. $P<0.05$ was considered significant throughout.

\section{RESULTS}

Feasibility in the clinical study

Sixteen subjects (six females) were randomly assigned to DCS or placebo. One subject assigned to placebo did not meet inclusion criteria due to accepting and swallowing all presentations without disruptions during structured meal protocol and was excluded. Ten children received DCS or placebo via gastrostomy tube and five by mouth (syringe, cup). There were no group differences on demographic or clinical measures (Table 1 ).

The study drug was delivered in 75 of 76 planned doses (39 DCS; 36 placebo). One subject randomized to BI+DCS discontinued participation after day 4 (12 meals) due to diarrhea and did not return for follow-up. For this subject, we carried the last set of observations forward from meal 12 to meal 15 to have complete data sets on all the 15 subjects. Otherwise, no adverse side effects were detected. All other subjects completed intervention and the 1-month follow-up visit.

\section{DCS facilitates BI for chronic feeding aversion}

Because outcome measures failed the Anderson-Darling test for normality, we rank-ordered the data before analyses. A significant main effect of meal was observed for rapid swallowing $\left(F_{(14,177)}=3.94, \quad P<0.001\right)$ and disruptions $\left(F_{(14,183)}=3.20\right.$, $P<0.001)$, indicating both groups showed significant improvement in swallowing food, with coinciding decreases in mealtime disruptions (Figures $1 \mathrm{a}-\mathrm{c}$ ). We next examined the pre/post treatment effects within each group. When comparing baseline and final day treatment meals (meals 1 and 15, respectively), $\mathrm{BI}$ +DCS substantially improved the median percentage of bites rapidly swallowed $\left(t_{(7)}=-8.95 ; P<0.001\right)$, whereas improvement in the $\mathrm{BI}+$ placebo group was not significant $\left(t_{(6)}=-2.29 ; P>0.1\right.$; Figure 1a). Highlighting rapid effects of DCS, there was marked improvement from meal 1 to meal 2 in the BI+DCS group in 
a

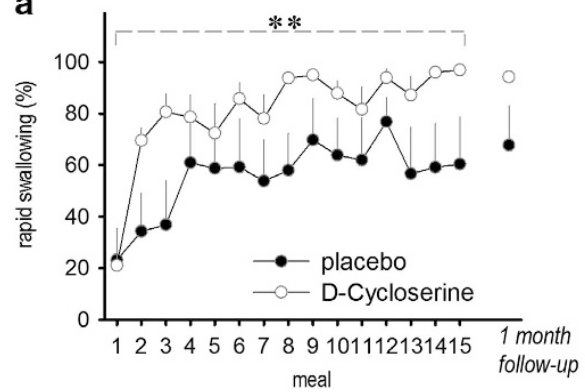

b

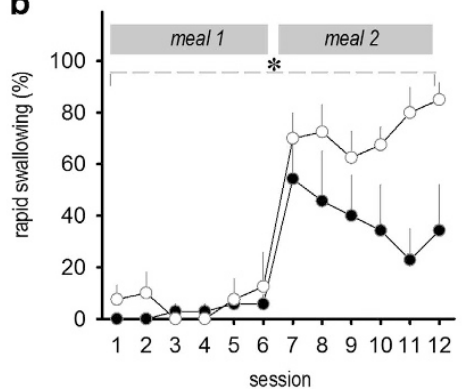

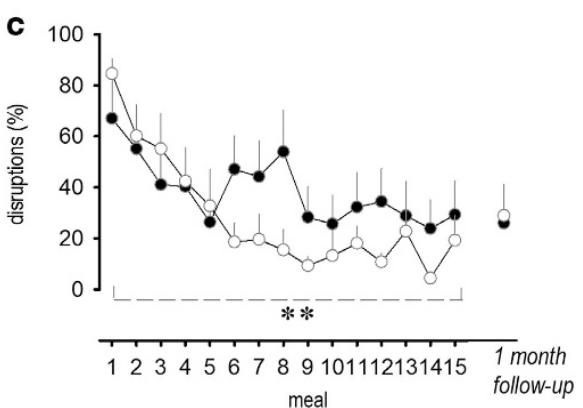

Figure 1. DCS successfully augments the treatment of severe feeding aversion in children. (a) Rapid swallowing is improved by $\mathrm{BI}$ in children with ARFID, and DCS augments this effect. (b) A comparison of individual feeding sessions from meals 1 and 2 highlights the rapid effect of DCS. (c) Mealtime disruptions were also reduced by $\mathrm{Bl}$, and this too was augmented by DCS. $n=7-8$ per group. Mean+s.e.m., $* P<0.05$; ${ }^{*} P<0.001$. ARFID, avoidant/restrictive food intake disorder; $\mathrm{Bl}$, behavioral intervention; $\mathrm{DCS}, \mathrm{D}$-cycloserine.

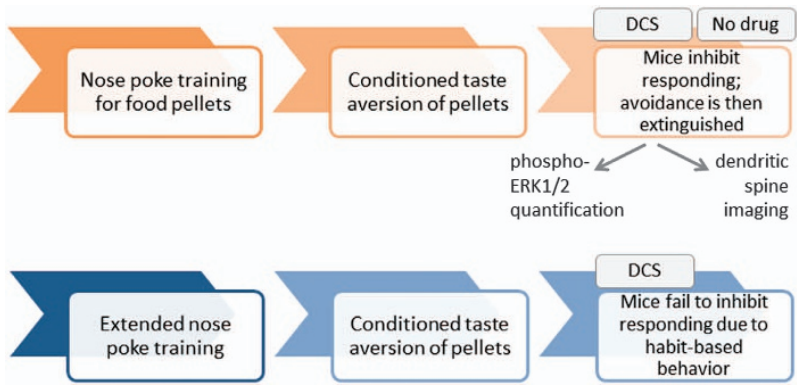

Figure 2. Timeline of experimental events in mice. (Top row) Mice were trained to nose poke for food pellets; conditioned aversion was then induced by pairing the food pellets with $\mathrm{LiCl}$, causing operant avoidance. We tested whether DCS could facilitate the extinction of this avoidance when mice were presented with pellets in the absence of $\mathrm{LiCl}$. DCS was administered following the first three extinction conditioning sessions, then the mice were further tested in the absence of drug to assess the long-term effects of DCS, if any. Phospho-ERK1/2 and dendritic spine imaging followed. (Bottom row) In our final experiment (Figure 5), the mice were extensively trained to develop nose-poking habits. Conditioned aversion was induced, then nose poking was quantified. DCS treatment accompanied these test sessions. DCS, D-cycloserine.

median percentage of bites rapidly swallowed (18.6-68.8\%; $\left.t_{(7)}=-4.94 ; P<0.005\right)$ compared with the BI+placebo arm (14.2$25.0 \% ; t_{(6)}=-0.43 ; P>0.9$; Figure $1 \mathrm{~b}$ ). Mealtime disruptions also decreased dramatically for those randomized to $\mathrm{BI}+\mathrm{DCS}\left(t_{(7)}=5.99\right.$; $P<0.005)$ compared with the BI+placebo group $\left(t_{(6)}=1.61 ; P>0.4\right.$; Figure $1 c)$. We also examined the number of meals elapsed before a study participant rapidly swallowed $\geqslant 80 \%$ of their bites for two meals. On average, the DCS group required 5.8 meals (s.d.: 2.6) to achieve this common clinical benchmark, whereas the placebo group required an average of 8.6 meals (s.d.: 6.1). In addition, all participants in the drug group had at least two meals in which $\geqslant 80 \%$ of bites were rapidly swallowed, whereas only four of seven subjects (57\%) in the placebo group achieved this level. No change in target behaviors from meal 15 to follow-up ( 1 month) was detected for either group, suggesting maintenance in treatment effects (Figure 1).

In summary, DCS rapidly facilitated the extinction of feeding aversions in children, increasing food acceptance and decreasing problem behavior.

Neurobiological insights via cross-species facilitation of avoidance extinction

In parallel, we developed a model of feeding aversion and avoidance extinction in mice. Timelines for our animal experiments are provided in Figure 2. We trained mice to nose poke for food reinforcers in operant conditioning chambers. Mice that would ultimately be designated to either vehicle-only (that is, placebo) or DCS did not differ in nose-poke rates $(P>0.5$; Figure 3a). The food reinforcer was then paired with $\mathrm{LiCl}$, inducing conditioned taste aversion, indicated by a decrease in free food consumption (main effect $F_{(2,18)}=10.5, P<0.005$; interaction $P>0.5$; Figure $3 b$ ). Accordingly, the mice also reduced nose poking for the food pellets when returned to the conditioning chambers (main effect $\mathrm{F}_{(1,9)}=19.8, P<0.005$; interaction $P>0.2$; Figure $3 c$ ). This test session served as a baseline against which the other sessions were normalized. Immediately following, the mice were injected with vehicle or DCS, $15 \mathrm{mg} \mathrm{kg}^{-1}$, a dose widely used in studies focused on conditioned fear extinction. ${ }^{11,33-36}$ DCS increased subsequent nose-poke rates, indicating the facilitation of avoidance extinction (main effect $F_{(1,9)}=5.6, P<0.05$; Figure $3 d$ ). Notably, the effects of DCS were detectable following the first treatment (Figure 3e, right), paralleling the rapid treatment effects observed in children (Figure 3e, left).

Next, we replicated this experiment using a 10-fold lower dose. The mice acquired the nose-poke response without group differences ( $P>0.5$; Figure $4 a)$. When the food reinforcer was paired with $\mathrm{LiCl}$, mice decreased consumption, indicating aversion ([main effect $F_{(1,19)}=52.5, P<0.001$; interaction $P>0.2$; Figure $4 b$ ). Accordingly, the mice also nose poked less in the operant conditioning chambers (main effect $\mathrm{F}_{(1,19)}=12.1, P<0.005$; interaction $F<1$; Figure 4c). DCS treatment again increased nose-poke rates, evidence of aversion extinction (main effect $F_{(1,19)}=4.3$, $P \leqslant 0.05$; Figure $4 d$ ).

We next assessed levels of phosphorylated ERK1/2, a putative intracellular mechanism of DCS action and a marker of synaptic activity, in frontal cortical brain regions involved in food-related decision making. Within the lateral frontal cortex (M2 and oPFC), DCS increased phospho-ERK1/2 (main effect of DCS $F_{(1,14)}=8.95$, $P<0.05$; no interaction $P>0.05$; Figure $4 \mathrm{e}$ ). Along the medial wall of the prefrontal cortex, however, no effect of DCS was detected (no main effect $P>0.4$, no interaction $P>0.1$; Figure $4 \mathrm{e}$ ).

To better understand neurobiological changes accompanying DCS-augmented treatment of food aversion, we also imaged and reconstructed dendritic spines in the oPFC (Figure 4f), which regulates food-related decision making. ${ }^{20}$ DCS did not modify the overall dendritic spine density or the densities of spines classified as stubby, mushroom or thin (all $P>0.4$; Figures $4 \mathrm{~g}$ and $\mathrm{h}$ ). However, DCS enlarged dendritic spine head diameters, indicated by a rightward shift in the cumulative density function representing spine head size (Kolmogorov-Smirnov $P<0.01$; Figure 4i). 

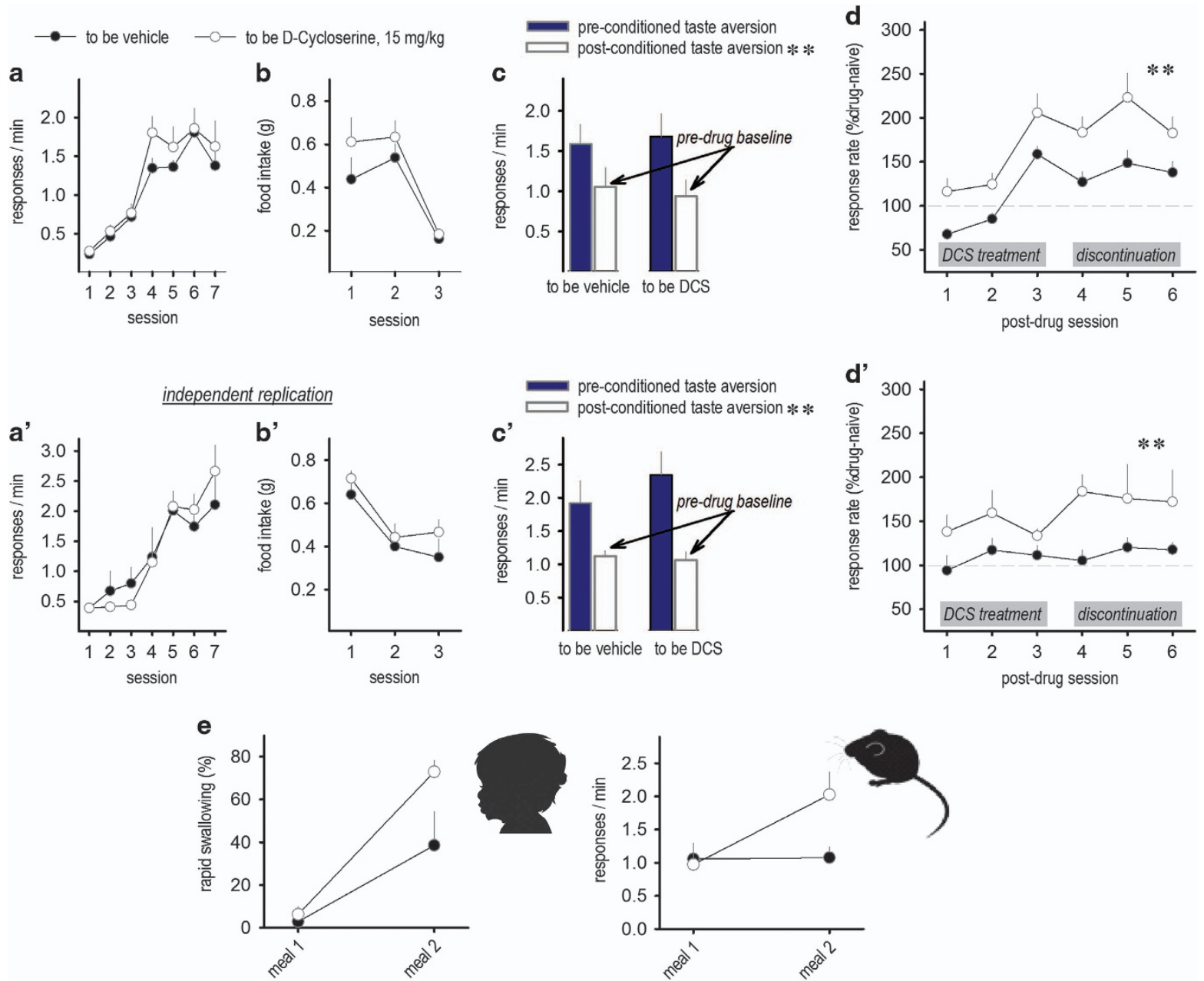

Figure 3. DCS enhances the extinction of conditioned food avoidance in mice. (a) The mice were trained to nose poke for food reinforcers. (b) Food reinforcers were paired with $\mathrm{LiCl}$, inducing temporary gastric malaise and reducing food consumption (that is, conditioned aversion). (c) Accordingly, nose-poke rates associated with the food pellets dropped when mice were returned to the conditioning chambers. Immediately following this extinction session, the mice were treated with vehicle or DCS. In other words, plots a-c represent naive animals, subsequently assigned to either vehicle (placebo) or DCS, plotted separately to confirm no differences in baseline acquisition of either the task or conditioned aversion. (d) DCS significantly enhanced food-reinforced nose-poke rates above extinction baseline (100\%, represented by the dashed gray line), and this persisted even when treatment was discontinued. $n=9$ per group. ( $\mathbf{a}^{\prime}-\mathbf{d}^{\prime}$ ) These represent an independent replication of the same effect, despite typical behavioral variabilities between independent cohorts. $n=10-11$ per group. (e) In both humans (left) and rodents (right), DCS acted rapidly, with group divergences by meal 2 (the first post-DCS session in the mouse, session 1 in $\mathbf{d}^{\prime}$ ). Mean +s.e.m., ${ }^{* *} P<0.00$. DCS, D-cycloserine.

DCS augments goal-directed response selection

To further test whether DCS facilitates the goal-directed decision making regarding whether to respond for food, we next used a nose-poke training protocol in mice that induces high rates of nose poking. We intended to induce stimulus-dependent habits, which counter goal-directed response strategies. This allowed us to determine whether DCS enhanced goal-directed response choice. This procedure effectively doubled the rate of poking (Figure $5 \mathrm{a}$ ) relative to earlier cohorts of animals (Figures 3a and 4a). By over-training food-seeking behavior in this manner-so that it assumed inflexible, habitual qualities, which are by definition insensitive to outcome value ${ }^{27,37}$ - we created an opportunity to test whether DCS treatment specifically facilitates goal-directed value-based action selection (that is, in contrast to nonspecific increases in feeding in general or to a reduction in cue-based associative conditioning).

As before, there were no differences during training between mice that were ultimately assigned to vehicle or DCS groups $(P>0.5$; Figure $5 a)$. We again paired the food reinforcers with $\mathrm{LiCl}$, reducing food intake (main effect $\mathrm{F}_{(2,16)}=36, P<0.001$; interaction $F_{(2,16)}=2.7, P>0.05$; Figure $\left.5 b\right)$. As expected, the mice failed to use the now-reduced value of the food reinforcer to guide response strategies when returned to the conditioning chambers: instead, the mice continued to generate robust response rates-behavioral habits (baseline vs post-conditioning $F_{(1,8)}=5.1, P>0.05$; Figure $5 c$ ). Subsequent DCS treatment reinstated goal-directed decision making, in this case reducing nose-poke rates associated with the devalued food pellet (interaction $\mathrm{F}_{(1,8)}=5.5, P<0.05$; Figure $5 \mathrm{~d}$ ). These findings suggest that DCS can enhance extinction conditioning by facilitating goal-directed learning and memory systems. Moreover, DCS-mediated facilitation of avoidance extinction cannot be attributed to nonspecific increases in feeding in general.

\section{DISCUSSION}

Here we believe we report the first randomized, double-blind, placebo-controlled trial examining DCS as an adjunct to BI for 

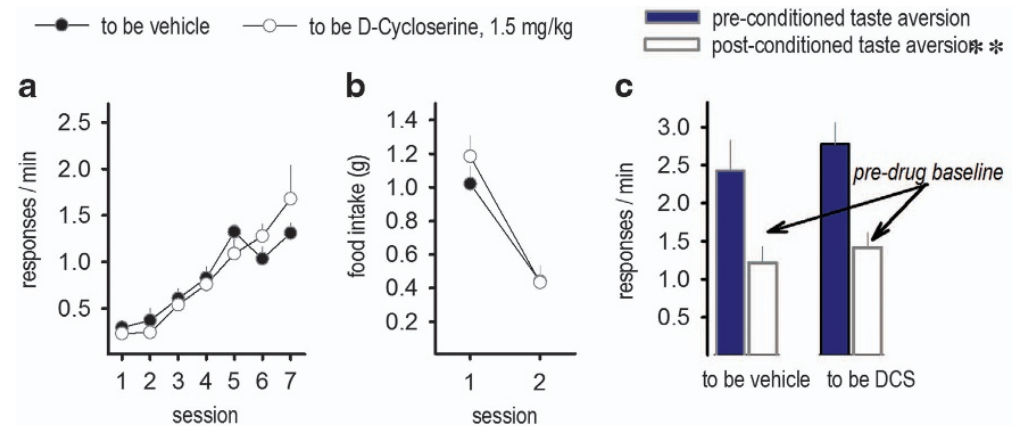

e

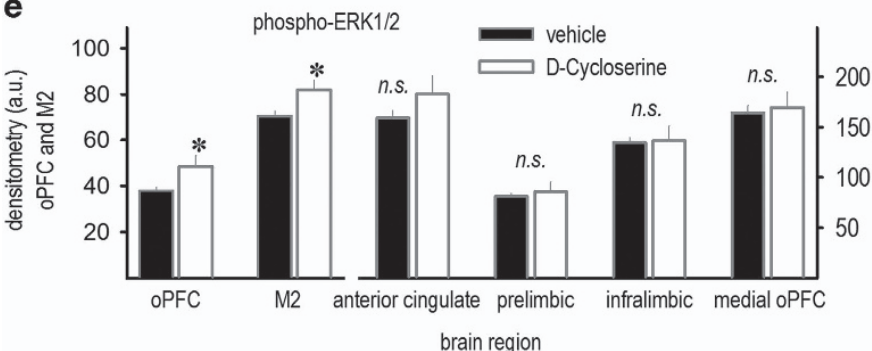

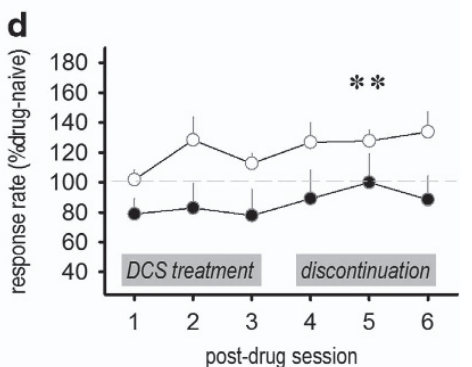

f

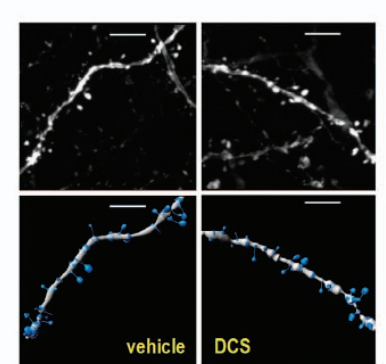

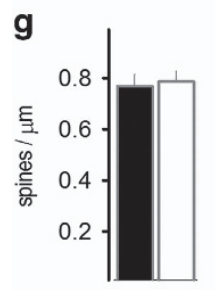
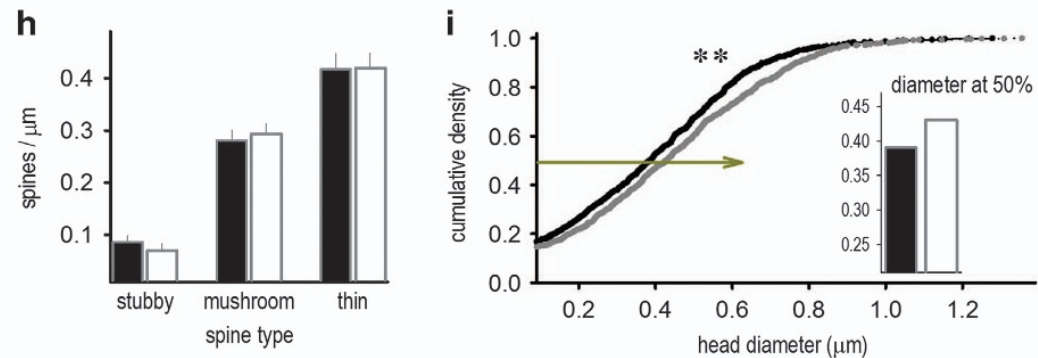

Figure 4. 'Low-dose' DCS enhances the extinction of conditioned food avoidance and induces ERK1/2 phosphorylation and structural plasticity. (a) Mice were trained to nose poke for food reinforcers, then (b) the food pellets were paired with LiCl, inducing conditioned aversion. (c) Accordingly, nose-poke rates associated with the food pellets also dropped when mice were returned to the conditioning chambers. Immediately following this first extinction session, the mice were treated with a 10-fold lower dose of DCS than in Figure.3 $\left(1.5 \mathrm{mg} \mathrm{kg}^{-1}\right)$. (d) DCS significantly enhanced food-reinforced nose-poke rates above extinction baseline (100\%, represented by the gray line), and this persisted even when treatment was discontinued, $n=7$ per group. (e) Immunostaining for phospho-ERK1/2 revealed higher levels following DCS in the oPFC and M2. Meanwhile, levels in the medial prefrontal cortex (anterior cingulate, prelimbic, infralimbic, medial oPFC) did not change, $n=6-10$ per group. (f) Dendritic spines in the oPFC were also imaged, enumerated and reconstructed. Representative dendritic branches and three-dimensional reconstructions are shown. (g) Overall densities did not differ, (h) nor did the subtypes of dendritic spines. (i) Spine head diameter, however, enlarged, suggesting that existing spines became larger in DCS-treated mice. Inset: at the 50th percentile, control spine heads were less than $0.4 \mu \mathrm{m}$ in diameter, whereas DCS-treated mice had larger heads, $>0.4 \mu \mathrm{m}$. Scale bar, $2 \mu \mathrm{m} . n=7$ per group. Mean+s.e.m., ${ }^{*} P<0.05 ;{ }^{* *} P<0.01$. DCS, D-cycloserine; NS, not significant.
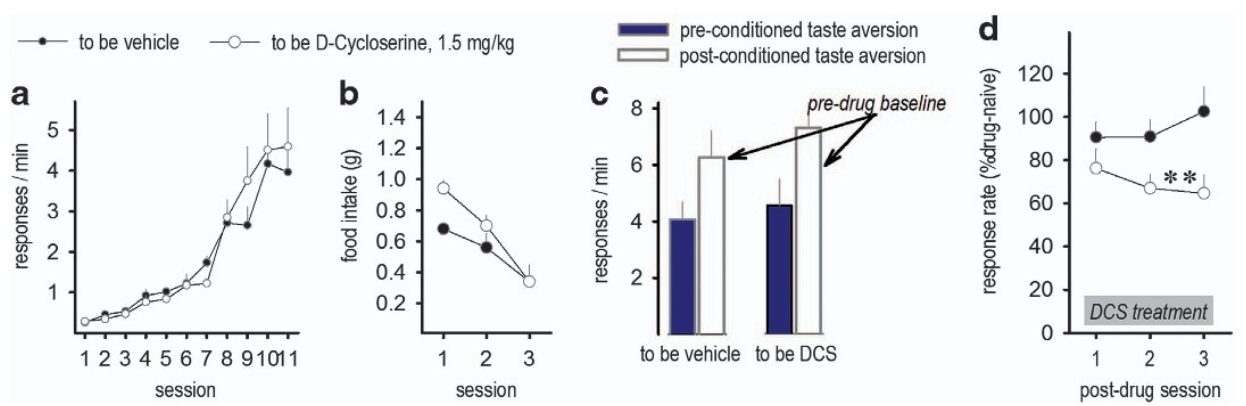

Figure 5. DCS enhances goal-directed response selection in mice. (a) Mice were trained to nose poke for food reinforcers using an extensive training protocol that generated high response rates. These protocols induce habit-based nose poking that is insensitive to conditioned food aversion, that is, inflexible habits. (b) We then paired the food pellets with $\mathrm{LiCl}$ in a separate environment, inducing conditioned aversion. (c) In accordance with the extended training protocol, nose-poke rates nevertheless continued to increase when mice were returned to the conditioning chambers $(P=0.053)$, despite conditioned food aversion, as expected. (d) In this case, DCS treatment reduced nose-poke rates, evidence that DCS enhances sensitivity to reinforcer value, $n=5$ per group. Mean+s.e.m., $* * P<0.001$, sessions $2-3$. DCS, D-cycloserine. 
chronic, severe food refusal. $\mathrm{BI}$ is an established treatment for chronic and severe food refusal. ${ }^{1,4,8,9}$ Not surprisingly, mealtime behaviors substantially improved for both the groups. However, DCS further enhanced response to intervention, rapidly increasing food acceptance, reducing disruptive behaviors and decreasing the number of meals required to achieve accepted clinical benchmarks. Further study could test whether DCS could permit a more timely elimination of, for example, supplemental feedings via feeding tube or bottle dependence in affected individuals, coupled by a decrease in problem behavior at mealtime, a significant source of stress for caregivers. Our parallel investigations in rodents aligned with human findings. Further, the augmentation of avoidance extinction was associated with structural plasticity of dendritic spines-the primary sites of excitatory plasticity in the brain-in the oPFC (Figure 4), a brain region intimately linked with food- and reward-related decision making.

Although small, our clinical study supports the feasibility of pairing DCS with BI. Enrollment and treatment occurred over a relatively brief period of time ( 5 months), and 14 of 15 children completed the trial. Further, our findings are consistent with other studies that also reported that low-dose DCS is well tolerated in pediatric populations. ${ }^{16}$ One child exited our study due to onset of diarrhea. Although not a commonly observed side effect, ${ }^{16}$ it should be considered possibly related to DCS. The contribution of dietary shifts or gastroenteritis should also be considered. Finally, our intervention targeted a sample of young children with pediatric feeding disorders. As such, our findings supporting further investigation of DCS as a treatment adjunct for pediatric feeding disorders may not be applicable to older children with avoidant/restrictive food intake disorder, which may involve a different etiology and treatment approach.

\section{Neurobiological insights via cross-species facilitation of food} refusal extinction

To provide potential insight into neurobiological mechanisms associated with successful intervention, we developed a model of feeding avoidance in mice to complement our clinical investigation. We first trained mice to nose poke for food reinforcers in operant conditioning chambers, then applied two distinct experimental manipulations: first, the food reinforcers were paired with $\mathrm{LiCl}$, inducing temporary gastric malaise and conditioned aversion. We selected this particular conditioned aversion approach to parallel a primary cause of aversions in our clinical population-pain and discomfort associated with consumption of food. In our mice, conditioned aversion reduced nose poking for the food reinforcer. This then allowed us to determine the effects of DCS on the extinction of avoidance. This experimental phase represents our animal corollary of a successful human BI. Paralleling effects in children, DCS decreased the time to extinction, with a broad (10-fold) effective dose range. Indeed, elevated food-reinforced response rates immediately followed the first treatment. The beneficial effects of DCS were also durable, persisting beyond the active treatment period.

As noted, DCS had a broad active dose range, up to $30 \mathrm{mg} \mathrm{kg}^{-1}$ in our pilot studies (not shown), and as low as $1.5 \mathrm{mg} \mathrm{kg}^{-1}$ (Figure 4), 10-fold lower than that typically used in rodents. ${ }^{11,33-36,38}$ The neurobehavioral consequences of drug doses at the low end of a given dose-response curve are most likely associated with a drug's primary mechanism of action, whereas higher doses increase the likelihood of off-target effects. This point is important for two reasons. First, it provides preclinical evidence for successful usage of low doses of DCS in clinical studies (an important consideration in all cases, but especially in pediatric populations). Second, it suggests specificity. Although DCS is an analog of glycine that has considerable activity at GluN2C-containing NMDA receptors, GluN2C has relatively low expression levels in the mature cortex..$^{39,40}$ DCS also has partial agonist effects at GluN2A-containing receptors. ${ }^{41}$ The facilitation of avoidance extinction following 'low-dose' DCS is thus more likely associated with GluN2A-mediated plasticity. As such, intracellular signaling mechanisms may include extracellular signal-regulated kinase $1 / 2($ ERK $1 / 2){ }^{42}$ preferentially stimulated by GluN2A- relative to GluN2B-containing NMDA receptors, and linked with extinction conditioning in multiple contexts. ${ }^{43}$

Based on these findings, we measured levels of the phosphorylated, active form of ERK1/2 following 'low-dose' DCS, revealing elevations in the oPFC and M2 (premotor cortex in mice, functionally akin to primate pre-supplementary motor area). This is significant because the oPFC is innervated by sensory structures, allowing this prefrontal cortical region to integrate sensory information into decision-making calculations and behavioral response strategies, ${ }^{20}$ and both the oPFC and $\mathrm{M} 2$ are involved in selecting actions based on anticipated food outcomes. ${ }^{44,45}$ Interestingly, low-dose DCS did not induce ERK1/2 phosphorylation in prefrontal cortical structures positioned along the medial wall, despite robust expression levels at baseline. These structures are associated with goal-directed food seeking (the prelimbic cortex ${ }^{46}$ ), effort-based decision making and planning (the anterior cingulate cortex and medial orbitofrontal $\operatorname{cortex}^{47}$ ), and extinction (the infralimbic cortex ${ }^{48,49}$ ). Although it is possible that other sampling time points (for example, closer to the time of behavioral testing) would have revealed phospho-ERK1/2 changes in these structures, ${ }^{50}$ why the lateral structures, the oPFC and M2, would be differentially sensitive to the effects of DCS on phospho-ERK1/2 is unclear. These findings are nevertheless provocative, given that ERK1/2 is essential for synaptic plasticity, ${ }^{51}$ and certain neurobiological systems, such as estradiol-mediated intracellular signaling, require ERK $1 / 2$ activation to modify dendritic spines, the primary sites of excitatory plasticity in the brain. ${ }^{52}$

We next imaged and reconstructed dendritic spines in the oPFC, again following 'low-dose' DCS $\left(1.5 \mathrm{mg} \mathrm{kg}^{-1}\right)$. Dendritic spine counts were unchanged, paralleling prior reports that higher doses of DCS (30-320 mg kg $\left.{ }^{-1}\right)$ have no effects on dendritic spine density/type in the hippocampus or medial prefrontal cortex. ${ }^{53}$ However, three-dimensional reconstruction revealed increased spine head diameters following DCS. This is significant because spine heads contain the postsynaptic machinery necessary for synaptic plasticity and intracellular signaling. ${ }^{54}$ Accordingly, we suggest that DCS induces plasticity in existing spines-rather than causing spinogenesis or spine pruning per se-and that these changes create a neural environment favorable to extinction. Alternatively, extinction conditioning itself could cause dendritic spine plasticity, which is then augmented by DCS. Because we did not include a no-extinction group here, future studies would be required to dissociate these possibilities. Additional investigations could also determine whether DCS modifies dendritic spine structures in other brain regions.

We imaged dendritic spines within a subregion of the oPFC, the ventrolateral region, which is necessary for conditioned fear extinction. ${ }^{55,56}$ Specifically, ventrolateral oPFC inactivation interferes with extinction conditioning, see also ref. 57 . The oPFC is conceptualized as allowing organisms to recognize the expectancy violation that occurs when a conditioned stimulus, like a tone, no longer predicts an outcome, like a foot shock, and to update expectations accordingly, hence failures in extinction when the oPFC is compromised. In the present experiments, oPFC activity could compare the expected outcome-either gastric malaise following food sampling (in our rodent model) or a prior history of feeding difficulties (in our treated children)-with the actual outcome-successful caloric intake accompanied by either no malaise for rodents or contacting the primary and secondary reinforcement associated with eating in children. As a result, expectancies would be updated, and extinction of feeding avoidance would occur. Dendritic spine head elaboration-as 
occurred with DCS treatment-and consequent synaptic strengthening within the oPFC could allow for more rapid updating of new associations, facilitating extinction. This model is consistent with the notion that food refusal in children with pediatric feeding disorders is maintained by escape from an aversive stimulus and is likely the result of conditioned food aversion that occurs from pairing unpleasant consequences (for example, pain, nausea and/ or fatigue) with eating. ${ }^{9}$

Within the oPFC, deep-layer dendrites are targeted by projections from the basolateral amygdala, ${ }^{21}$ which encodes responsereward contingencies, ${ }^{58,59}$ potentially via interactions with the oPFC $^{60}$ (but see ref. 46). Supporting the possibility that DCS facilitates plasticity in a basolateral amygdala-OPFC circuit, we also report that DCS enhanced goal-directed decision making. In this case, food-seeking behaviors were overtrained such that they assumed inflexible habitual qualities that are, by definition, insensitive to outcome value. ${ }^{37}$ DCS treatment brought goaldirected value-based action selection, which is basolateral amygdala-dependent, ${ }^{58-61}$ back 'on-line' (in this case, decreasing food-seeking behavior).

Although we focused here on the oPFC, it seems plausible that the central and basal nuclei of the amygdala, the gustatory thalamus, and the insular cortex also have roles in the development and extinction of feeding avoidance, ${ }^{62,63}$ and interestingly, the neuroplasticity-associated neurotrophin brainderived neurotrophic factor is involved in both aversion- and reward-based extinction and goal-directed response choice, ${ }^{63-65}$ providing another potential pharmacotherapeutic target in future studies.

In conclusion, goal-directed action likely has a key role in overcoming avoidance and in learning to consume food when it no longer has adverse consequences, for example, after resolution of an underlying medical concern, when untreated children might otherwise maintain food refusal and incur severe consequences of protracted feeding disorders. ${ }^{1-9}$ Overall, the current results suggest that DCS can facilitate extinction, resulting in decreased time to effect and overall food acceptance. In tandem with larger clinical trials verifying the benefits of DCS in the treatment of avoidant/restrictive food intake disorder, future investigations should crystallize the molecular- and circuit-level mechanisms that regulate avoidance extinction and goal-directed food seeking, behaviors that must be maintained in order for feeding aversions to be ameliorated.

\section{CONFLICT OF INTEREST}

WGS has received research support and served as a consultant for Nutricia. LS has served as a consultant for the following companies: Roche, Coronado, Bracket, Neuren, MedAdvante. LS also received research support from Shire and Roche. The remaining authors declare no conflict of interest.

\section{ACKNOWLEDGMENTS}

This work was supported by an award from the Emory/Children's Pediatric Research Center \& Yerkes National Primate Research Center. The Yerkes National Primate Research Center is supported by the Office of Research Infrastructure Programs/OD P51OD011132. The Emory Microscopy Core is supported by an NINDS Core Facilities grant, P30NS055077. We thank Drs Kerry Ressler and Warren Jones for their guidance and Savonya McAllister, Ashley Trumbull, and Emily Maddox for assisting with conducting structured meals and helping with data collection.

\section{REFERENCES}

1 Sharp WG, Jaquess DL, Morton JS, Herzinger C. Pediatric feeding disorders: a quantitative synthesis of treatment outcomes. Clin Child Fam Psychol Rev 2011; 13: 348-365.

2 American Psychiatric Association. Diagnostic and Statistical Manual of Mental Disorders, 5th edn. American Psychiatric Publishing: Washington, DC, USA, 2013.
3 Satter E. The feeding relationship: problems and interventions. J Pediatr 1990; 117 (2 Pt 2): S181-S189.

4 Kerwin ME. Empirically supported treatments in pediatric psychology: severe feeding problems. J Pediatr Psychol 1999; 24: 193-214.

5 Volkert VM, Piazza CC. Pediatric feeding disorders. In: Sturmey P, Hersen M (eds). The Handbook of Evidence Based Practice in Clinical Psychology, Volume 1, Child and Adolescent Disorders. JohnWiley \& Sons: New York, NY, USA, 2012, pp 456-481.

6 Babbitt RL, Hoch TA, Coe DA, Cataldo MF, Kelly KJ, Stackhouse C et al. Behavioral assessment and treatment of pediatric feeding disorders. J Dev Behav Pediatr 1994; 15: 278-291.

7 Piazza CC, Fisher WW, Brown KA, Shore BA, Patel MR, Katz RM et al. Functional analysis of inappropriate mealtime behaviors. J Appl Behav Anal 2003; 36: 187-204.

8 Lukens $\mathrm{CT}$, Silverman $\mathrm{AH}$. Systematic review of psychological interventions for pediatric feeding problems. J Pediatr Psychol 2014; 38: 903-917.

9 Sharp WG, Volkert VM, Scahill L, McCracken CE, McElhanon BM. A systematic review and meta-analysis of intensive multidisciplinary intervention for pediatric feeding disorders: how standard is the standard of care? J Pediatr 2017; 181: 116-124.

10 Williams KE, Riegel K, Gibbons B, Field DG. Intensive behavioral treatment for severe feeding problems: a cost-effective alternative to tube feedings? J Dev Phys Disabil 2007; 19: 227-235.

11 Walker DL, Ressler KJ, Lu KT, Davis M. Facilitation of conditioned fear extinction by systemic administration or intra-amygdala infusions of D-cycloserine as assessed with fear-potentiated startle in rats. J Neurosci 2002; 22: 2343-2351.

12 Hofmann SG, Meuret AE, Smits JA, Simon NM, Pollack MH, Eisenmenger $\mathrm{K}$ et al. Augmentation of exposure therapy with D-cycloserine for social anxiety disorder. Arch Gen Psychiatry 2006; 63: 298-304.

13 Ressler KJ, Rothbaum BO, Tannenbaum L, Anderson P, Graap K, Zimand E et al. Cognitive enhancers as adjuncts to psychotherapy: use of D-cycloserine in phobic individuals to facilitate extinction of fear. Arch Gen Psychiatry 2004; 61: 1136-1144.

14 Wilhelm S, Buhlmann U, Tolin DF, Meunier SA, Pearlson GD, Reese HE et al. Dcycloserine augmentation of behavior therapy for obsessive compulsive disorder. Am J Psychiatry 2008; 165: 335-341.

15 Otto MW, Tolin DF, Simon NM, Pearlson GD, Basden S, Meunier SA et al. Efficacy of d-cycloserine for enhancing response to cognitive-behavior therapy for panic disorder. Biol Psychiatry 2010; 67: 365-370.

16 Storch EA, McKay D, Reid JM, Geller DA, Goodman WK, Lewin AB et al. Dcycloserine augmentation of cognitive-behavioral therapy: Directions for pilot research in pediatric obsessive-compulsive disorder. Child Youth Care Forum 2010; 39: 101-112.

17 Norberg MM, Krystal JH, Tolin DF. A meta-analysis of D-cycloserine and the facilitation of fear extinction and exposure therapy. Biol Psychiatry 2008; 63: 1118-1126.

18 Bowers ME, Ressler KJ. An overview of translationally informed treatments for posttraumatic stress disorder: animal models of pavlovian fear conditioning to human clinical trials. Biol Psychiatry 2015; 78: E15-E27.

19 Smits J, Rosenfield D, Otto MW, Powers MB, Hofmann SG, Telch MJ et al. DCycloserine enhancement of fear extinction is specific to successful exposure sessions: evidence from the treatment of height phobia. Biol Psychiatry 2013; 73: 1054-1058.

20 Grabenhorst F, Rolls ET. Value, pleasure and choice in the ventral prefrontal cortex. Trends Cogn Sci 2011; 15: 56-67.

$21 \mathrm{Kita} \mathrm{H}$, Kitai ST. Amygdaloid projections to the frontal cortex and the striatum in the rat. J Comp Neurol 1990; 298: 40-49.

22 Sharp WG, Stubbs KH, Adams H, Wells BM, Lesack RS, Criado KK et al. Intensive manual-based intervention for pediatric feeding disorders: Results from a randomized pilot trial. J Pediatr Gastroenterol Nutr 2016; 62: 658-667.

23 Ahearn $\mathrm{WH}$, Kerwin ME, Eicher $\mathrm{P}$, Shantz J, Swearingin W. An alternating treatments comparison of two intensive interventions for food refusal. J Appl Behav Anal 1996; 29: 321-332.

24 Sharp WG, Jaquess D, Bogart J, Morton J. Additive, multi-component treatment of emerging refusal topographies in a pediatric feeding disorder. Child Fam Behav Ther 2010; 32: 51-69.

25 Viera AJ, Garrett JM. Understanding interobserver agreement: the Kappa statistic. Fam Med 2005; 37: 360-363.

26 Feng G, Mellor RH, Bernsein M, Keller-Peck C, Nguyen QT, Wallace M et al. Imaging neuronal subsets in transgenic mice expressing multiple spectral variants of GFP. Neuron 2000; 28: 41-51.

27 Dickinson A, Nicholas DJ, Adams CD. The effect of the instrumental training contingency on susceptibility to reinforcer devaluation. Q J Exp Psychol 1983; 35B: 35-51.

28 Quinn JJ, Hitchcott PK, Umeda EA, Arnold AP, Taylor JR. Sex chromosome complement regulates habit formation. Nat Neurosci 2007; 10: 1398-1400. 
29 Franklin KBJ, Paxinos G. The Mouse Brain in Stereotaxic Coordinates, 3rd edn. Academic Press: New York, New York, 2007.

30 Gourley SL, Swanson AM, Koleske AJ. Corticosteroid-induced neural remodeling predicts behavioral vulnerability and resilience. J Neurosci 2013; 33: 11811-11816.

31 Radley JJ, Anderson RM, Hamilton BA, Alcock JA, Romig-Martin SA. Chronic stressinduced alterations in dendritic spine subtypes predict functional decrements in an hypothalamic-pituitary-adrenal-inhibitory prefrontal circuit. J Neurosci 2013; 33: 14379-14391.

32 Kolb B, Cioe J, Comeau W. Contrasting effects of motor and visual spatial learning tasks on dendritic arborization and spine density in rats. Neurobiol Learn Mem 2008; 90: 295-300.

33 Ledgerwood L, Richardson R, Cranney J. Effects of D-Cycloserine on extinction of conditioned freezing. Behav Neurosci 2003; 117: 341-349.

34 Ledgerwood L, Richardson R, Cranney J. D-Cycloserine and the facilitation of extinction of conditioned fear: consequences for reinstatement. Behav Neurosci 2004; 118: 505-513.

35 Ledgerwood L, Richardson R, Cranney J. D-cycloserine facilitates extinction of learned fear: effects on reacquisition and generalized extinction. Biol Psychiatry 2005; 57: 841-847.

36 Weber M, Hart J, Richardson R. Effects of D-cycloserine on extinction of learned fear to an olfactory cue. Neurobiol Learn Mem 2007; 87: 476-482.

37 Balleine BW, O'Doherty JP. Human and rodent homologies in action control: corticostriatal determinants of goal-directed and habitual action. Neuropsychopharmacology 2010; 35: 48-69.

38 Thanos PK, Subrize M, Lui W, Puca Z, Ananth M, Michzelides M et al. D-cycloserine facilitates extinction of cocaine self-administration in C57 mice. Synapse 2011; 65: 1099-1104.

39 Traynelis SF, Wollmuth LP, McBain CJ, Menniti FS, Vance KM, Ogden KK et al. Glutamate receptor ion channels: structure, regulation, and function. Pharmacol Rev 2010; 62: 405-496.

40 Monyer H, Burnashev N, Laurie DJ, Sakmann B, Seeburg PH. Developmental and regional expression in the rat brain and functional properties of four NMDA receptors. Neuron 1994; 12: 529-540.

41 Sheinin A, Shavit S, Benveniste M. Subunit specificity and mechanism of action of NMDA partial agonist D-cycloserine. Neuropharmacology 2001; 41: 151-158.

42 Gao C, Gill MB, Tronson NC, Guedea AL, Guzman YF, Huh KH et al. Hippocampal NMDA receptor subunits differentially regulate fear memory formation and neuronal signal propagation. Hippocampus 2010; 20: 1072-1082.

43 Cestagi V, Rossi-Arnaud C, Saraulli D, Costanzi M. The MAP(K) of fear: from memory consolidation to memory extinction. Brain Res Bull 2014; 105: 8-16.

44 Swanson AM, Allen AG, Shapiro LP, Gourley SL. GABAAa1-mediated plasticity in the orbitofrontal cortex regulates context-dependent action selection. Neuropsychopharmacology 2015; 40: 1027-1036.

45 Gremel CM, Costa RM. Orbitofrontal and striatal circuits dynamically encode the shift between goal-directed and habitual actions. Nat Commun 2013; 4: 2264.

46 Hart G, Leung BK, Balleine BW. Dorsal and ventral streams: the distinct role of striatal subregions in the acquisition and performance of goal-directed action. Neurobiol Learn Mem 2014; 108: 104-118.

47 Bradfield LA, Dezfouli A, van Holstein M, Chieng B, Balleine BW. Medial orbitofrontal cortex mediates outcome retrieval in partially observable task situations. Neuron 2015; 88: 1268-1280.

48 Peters J, Kalivas PW, Quirk GJ. Extinction circuits for fear and addiction overalap in prefrontal cortex. Learn Mem 2009; 16: 279-288.

49 Gourley SL, Taylor JR. Going and stopping: dichotomies in behavioral control by the prefrontal cortex. Nat Neurosci 2016; 19: 656-664.

50 Hart G, Balleine BW. Consolidation of goal-directed action depends on MAPK/ERK signaling in rodent prelimbic cortex. J Neurosci 2016; 36: 11974-11986.
51 English JD, Sweatt JD. A requirement for the mitogen-activated protein kinase cascade in hippocampal long term potentiation. J Biol Chem 1997; 272 19103-19106.

52 Tuscher JJ, Luine V, Fankfurt M, Frick KM. Estradiol-mediated spine changes in the dorsal hippocampus and medial prefrontal cortex of ovariectomized female mice depend on ERK and mTOR activation in the dorsal hippocampus. J Neurosci 2016; 36: 1483-1489.

53 Gupta SC, Yadav R, Pavuluri R, Morley BJ, Stairs DJ, Dravid SM. Essential role of GluD1 in dendritic spine development and GluN2B to GluN2A NMDAR subunit switch in the cortex and hippocampus reveals ability of GluN2B inhibition in correcting hyperconnectivity. Neuropharmacology 2015; 93: 274-284.

54 Pontrello CG, Ethell IM. Accelerators, brakes, and gears of actin dynamics in dendritic spines. Open Neurosci J 2009; 3: 67-86.

55 Zelinski EL, Hong NS, Tyndall AV, Halsall B, McDonald RJ. Prefrontal cortical contributions during discriminative fear conditioning, extinction, and spontaneous recovery in rats. Exp Brain Res 2010; 203: 285-297.

56 Zimmermann KS, Li CC, Rainnie DG, Ressler KJ, Gourley SL. Memory retention involves the ventrolateral orbitofrontal cortex: comparisons with the basolateral amygdale (revision in review).

57 Panayi MC, Killcross S. Orbitofrontal cortex inactivation impairs between- but not within-session Pavlovian extinction: an associative analysis. Neurobiol Learn Mem 2014; 108: 78-87.

58 Wellman LL, Gale K, Malkova L. GABAA-mediated inhibition of basolateral amygdala blocks reward devaluation in macaques. J Neurosci 2005; 25: 4577-4586.

59 Parkes SL, Balleine BW. Incentive memory: evidence the basolateral amygdala encodes and the insular cortex retrieves outcome values to guide choice between goal-directed actions. J Neurosci 2013; 33: 8753-8763.

60 Holland PC, Gallagher M. Amygdala-frontal interactions and reward expectancy. Curr Opin Neurobiol 2004; 14: 148-155.

61 West EA, Forcelli PA, Murnen AT, McCue DL, Gale K, Malkova L. Transient inactivation of basolateral amygdala during selective satiation disrupts reinforcer devaluation in rats. Behav Neurosci 2012; 126: 563-574.

62 Lin JY, Roman C, Arthurs J, Reilly S. Taste neophobia and C-Fos expression in the rat brain. Brain Res 2012; 1448: 82-88.

63 Rodriguez-Serrano LM, Ramirez-Leon B, Rodriguez-Duran LF, Escobar ML. Acute infusion of brain-derived neurotrophic factor in the insular cortex promotes conditioned taste aversion extinction. Neurobiol Learn Mem 2014; 116: 139-144.

64 Zimmermann KS, Yamin JA, Rainnie DG, Ressler KJ, Gourley SL. Connections of the mouse orbitofrontal cortex and regulation of goal-directed action selection by BDNF-TrkB. Biol Psychiatry 2017; 81: 366-377.

65 Gourley SL, Olevska A, Zimmermann KS, Ressler KJ, DiLeone RJ, Taylor JR. The orbitofrontal cortex regulates outcome-based decision-making via the lateral striatum. Eur J Neurosci 2013; 38: 2382-2388.

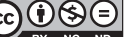

This work is licensed under a Creative Commons AttributionNonCommercial-NoDerivs 4.0 International License. The images or other third party material in this article are included in the article's Creative Commons license, unless indicated otherwise in the credit line; if the material is not included under the Creative Commons license, users will need to obtain permission from the license holder to reproduce the material. To view a copy of this license, visit http:// creativecommons.org/licenses/by-nc-nd/4.0/

(c) The Author(s) 2017 